\title{
Control of Upper Limb Active Prosthesis Using Surface Electromyography
}

\author{
Muhammad Asim Waris, Mohsin Jamil, Syed Omer Gilani, Yasar Ayaz \\ School of Mechanical \& Manufacturing Engineering (SMME) \\ National University of Sciences \& Technology (NUST) \\ Islamabad, Pakistan \\ asimwaris_6680@yahoo.com,mohsin@smme.nust.edu.pk,omer@smme.nust.edu.pk, yasar@smme.nust.edu.pk
}

Received: July 5, 2019. Revised: August 7, 2021. Accepted: September 14, 2021. Published: November 27, 2021.

\begin{abstract}
Electromyographic prosthesis with higher degrees of freedom is an expanding area of research. In this paper, active prosthesis with four degrees of freedom has been investigated, which can be used to fit a limb with amputation below elbow. The system comprises of multichannel inputs which correspond to the flexion and extension as well as supination and pronation. To find maximum surface neural activity, accurate placement of electrodes has been carried out on 10 subjects aged between 22-30 years. Signals (0-500 hertz) acquired from contracting voluntary muscles with minimum cross talk and common mode noise. Clean filtered EMG signal is then amplified precisely. Finally digitization is being done to drive bionic hand. Practical demonstration on a simple DC motor proved providential using this method for the two motions of an actual human arm. EMG Signals emanating from muscles dedicated to individual fingers have been recorded. Moreover modern classifiers; KNN and NN have been investigated carefully with selected features through different time and noise levels.
\end{abstract}

Keywords- Electromyography (EMG), flexion, extension, amplification, supination, pronation, KNN (K nearest neighbor), NN (neural network).

\section{INTRODUCTION}

The field of study that deals with the detection (from needle, cup and surface electrodes), analysis (from picoscope) and the use of electrical signals (for active prosthesis) is known as Electromayography (EMG). It involves techniques for analysis and recording the surface activity which produce electric potential by skeletal muscles [1]. The device which records EMG signals is called electromyograph. An electromayograph detects the electrical potential generated by muscle cells when these are contracted or neurologically activated [2].

To develop innovative upper limb prosthesis, the real challenge is to incorporate the device with intuitive, intelligent and human like system without losing its functionality having high deftness [3]. EMG surface activity stimulated by voluntary contraction of the targeted muscle considered as the intention of the mayo-electric prosthesis user [4]. Most commonly Mean Absolute Value (MAV) is used to determine the intension of the user, in which the absolute value of EMG signal is compared with predetermined threshold value.

Human body consists of muscles, composed of fibers having motor points on it. These points when activated generate motor point active potential. A motor unit (MU) is defined as an anterior horn cell, its axon and the muscle fibers innervated by the motor neuron [5]. Motor unit action potential (MUAP) is a train of pulses or summation of a group of muscle fiber action potential (MFAP) where superimposed information of muscle and generated pulses is determined by each (MFAP).As long as force is maintained or even increased motor unit generates pulses continuously and resultantly muscle contracts [6]. Motor points remain activated as long as muscles are being contracted, This continuous activation of motor points superimpose to form EMG signal, when muscle exerts more force greater number of motor points are activated , so we can conclude that lifting a heavy weight fires more motor points that lifting a lighter weight.

There are many factors which affect the EMG signals emanating from the muscles, important aspects are the type of muscle contraction that is occurring and the type of electrode used for the detection of these signals, Judicious application of recognized basis which can accurately identify the innervation zone with bounding effects of noise and cross talk providing immotility in signal and normalization of its amplitude which further enhance the single by removing the effect of many other variables [7]. EMG signal emanated can be used as a symptom about: contraction of muscle, force produced when muscle is being activated and tell us the fatigue variable of the targeted muscle.

Advance electronics and micro controller based designs brought new revolution is active prosthesis giving more degree of freedom to the designer. More movements and muscle activation can be controlled with the help of filter algorithms giving more functionality and maneuverability to the user.

\section{MEASURING DEVICES}

EMG due to its vast biomedical application becomes an interest of many researches working in the field of prosthetics [8]. Non-invasive techniques are the most desirable and applicable technique for measuring EMG signals with help of electrode. Which can be categorized in dry and gel type electrodes [9].

Gelled electrode contains gel between skin and the measuring electrode. These gelled electrodes are mostly disposable and not very feasible as it cannot be used for longer periods of time. Dry electrodes do not contain such medium 
and can be used for longer periods of time, which are ideal for active prosthesis.

Dry electrodes are further divided in to two types: active and passive. Passive electrodes do not require energy or current for their activation. Active electrodes require energy or current for their activation, these electrodes often having high input impedance and pre-amplification circuitry attached to it. It's important to prepare test subjects properly before testing [10]. Skin surface should be clean, hairs should be removed to avoid artifacts and electrode should be stationed properly holding its place. Gelled electrodes were used in this research with self-designed circuitry.

\section{SIGNAL ACQUISTION AND POSITIONING OF EMG ELECTRODES}

Positioning of EMG electrode and acquisition of required signal is an important feature of active prosthesis. Gelled electrodes which were used in the study were carefully place on the belly of the muscle, each electrode placed 1-2 cm from each other. The established location of electrodes is between innervation zone and the tendinous insertion [11].

Noise reduction is done for further enhancement of the acquired signal then this signal is send to an instrumentation amplifier for amplification purposes. Referenced signal must be acquired, which is isolated electrically and must be distanced from targeted muscle, the most desirable place for reference electrode is the neck or it should be unrelated to the muscles of forearm.

Placement of electrodes on the targeted muscles is an important part; small difference from the motor point can bring drastic effect on the amplitude of the signal. For flexion of hand; flexor digitorum profundus, which fans out in to four tendons connected to each of four fingers except thumb, is preferred muscle for the placement of electrode and for the extension, it should be on extensor digitorium communis. Individual signals from each finger have also been observed by placing electrode on the associated muscle of each finger.

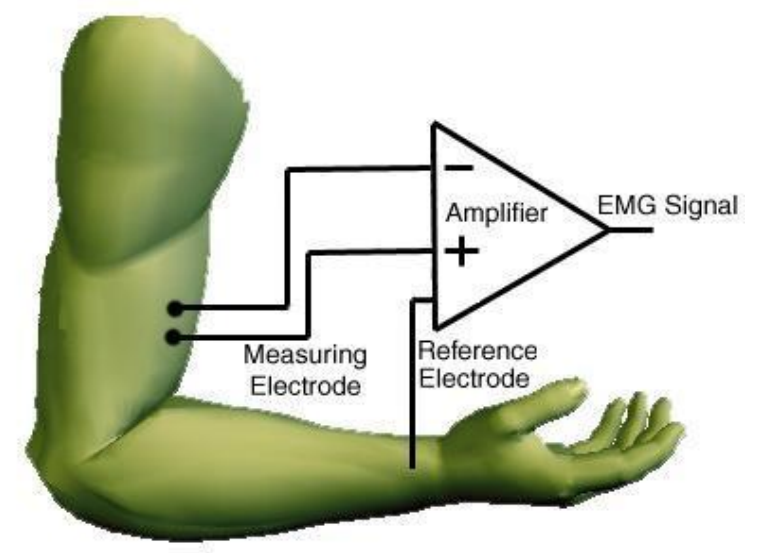

Figure1. General placement of surface EMG and reference electrodes.

For flexion of thumb, preferred location of electrode is on flexor pollicis longus, for its extension electrode should be placed on extensor pollicis longus. In case of pinkie, middle and ring finger electrode should be placed precisely on flexor carpi ulinaris, flexor carpi radialis and flexor palmaris longus respectively. Similarly for the extension of these fingers, electrode should be on extensor carpi ulinaris.

Index finger which is the most dexterous and sensitive finger controlling several motions of hand, for its flexion, flexor digitorum superficialis is the preferred location of electrode placement and for extension, recommended muscle is extensor inidcis.

\section{NOISE REDUCTION TECHNIQUE}

EMG signal has very low signal to noise (SNR) ratio, many factor bring about these disturbances, and one of the major portion of these noises are cardiac artifacts or Electrocardiography (ECG). Non-stationary nature of EMG signals keeps the amplitude ratio between EMG signals and cardiac artifacts variable.

A signal processing technique based on finite impulse response (FIR) adapter filter can be employed to reduce noise in which multi-electrode array is used for signal acquisition purposes [12]. In this method referencing is done with respect to ECG signals then adaptive filter is applied to reduce power line disturbances.

\section{A. Noise Reference Estimation}

Acquired signal $\mathrm{Z}(\mathrm{t})$ at the surface of the skin composed of EMG signal $n_{i}(t)$ and ECG signal $s_{i}(t)$. Band filter $(20 \mathrm{~Hz}$ : $40 \mathrm{~Hz}$ ) is then applied to the summation of the signal.

$$
\begin{aligned}
& Z(t)=\sum_{i=1}^{N}\left[n_{i}(t)+s_{i}(t)\right] \\
& \left.Z(t)=\sum_{i=1}^{N} n_{i}(t)+\sum_{i=1}^{N} s_{i}(t)\right]
\end{aligned}
$$

Where

$n_{i}(t)=$ Acquired Electromyographic $(\mathrm{EMG})$ signal

$s_{i}(t)=$ Acquired Electrocardiographic (ECG) signal

$\mathrm{N}=$ Electrodes attached to limb

Autocorrelation function is given as

Putting equation 1 in 2

$$
\begin{gathered}
\Gamma_{z z}(\tau)=E[Z(t) Z(t-\tau)] \\
\Gamma_{z z}(\tau)=E\left\{\left\lfloor\sum_{i=1}^{N} n_{i}(t)+\sum_{i=1}^{N} s_{i}(t) \| \sum_{i=1}^{N} n_{i}(t-\tau)+\sum_{i=1}^{N} s_{i}(t-\tau)\right\rfloor\right\} \\
\Gamma_{z z}(\tau)=\sum_{l, m}^{N} \Gamma_{n_{l} n_{m}}(\tau)+\sum_{l, m}^{N} \Gamma_{n_{l} s_{m}}(\tau)+\sum_{l, m}^{N} \Gamma_{n_{m} s_{l}}(\tau)+\sum_{l, m}^{N} \Gamma_{s_{l} s_{m}}(\tau)
\end{gathered}
$$

As ECG and EMG signal has no co-relation so above equation reduces.

$$
\Gamma_{z z}(\tau)=\sum_{l, m}^{N} \Gamma_{n_{l} n_{m}}(\tau)+\sum_{l, m}^{N} \Gamma_{s_{l} s_{m}}(\tau)
$$

High correlation of ECG signal at electrode channel yields $l \neq m$ 


$$
\begin{aligned}
& \Gamma_{z z}(\tau)=\sum_{i=1}^{N} \Gamma_{n_{l} n_{l}}(\tau)+\sum_{l, m}^{N} \Gamma_{n_{l} n_{m}}(\tau)+N^{2} \Gamma_{s s}(\tau) \\
& \Gamma_{z z}(\tau)=N^{2} \Gamma_{s s}(\tau)+N \Gamma_{n n}(\tau)+\sum_{l, m}^{N} \Gamma_{n_{l} n_{m}}(\tau)
\end{aligned}
$$

The above equation proves that the signal to noise ratio increase $\mathrm{N}$ times greater if we use array of electrode for the acquisition of EMG signals.

\section{B. Adaptive Filtering}

Power line interference is one of the major causes which decrease the quality of EMG signal significantly [13]. Adaptive filter can be really helpful in attenuating these interferences.

Raw EMG signal contains power line interferences under $60 \mathrm{~Hz}$. If we know the characteristic of noise, a filter can be designed to reduce that noise with high efficiency [14].

$$
y(k)=x(k)+\stackrel{\Delta}{n}(k)=s(k)+\lfloor n(k)-\stackrel{\Delta}{n}(k)\rfloor
$$

Where

$y(k)=$ Output signal of noise canceler

$x(k)=$ Input raw signal

$\Lambda$

$n(k)=$ Noise estimate

$n(k)=$ Noise influence on input raw signal $x(k)$

With the help of noise estimate, noise influence on the acquired signal can be minimized; the estimate noise can be deduced by finite impulse response (FIR) adaptive filter through which a sample of noise is given as input to the controller.

$\hat{n}(k)=\sum_{i=0}^{N-1} w_{i}(k) . r(k-i)$

$N-1=$ Order of the filter

$w_{i}(k)=$ Adjustable filter coefficients

$r(k)=$ Sample of noise or reference input

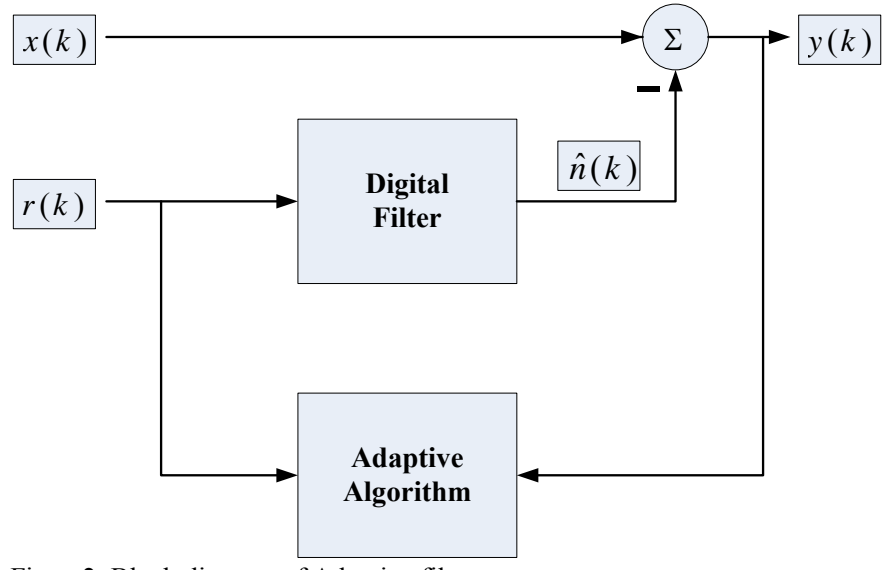

Figure2. Block diagram of Adaptive filter

\section{MODERN CLASSIFICATION TECHNIQUES}

After the amplification, classification of these EMG signals is done to identify the user's intended motion. Many authors investigated these classification strategies Sebelius et al. [15] and Paul et al. [16] are one of those who faced and investigated the issue of real-time implementation of artificial active prosthesis. Segmentation, in accordance with a flexion of the dedicated muscle, Pattern recognition, Feature extraction, Classification of signals and simulating actual prosthesis were the main subject of issue during the study [17]. Here Neural Network and K nearest neighbor classifiers have been studied.

\section{A. Neural Network Classifier}

In the recent past most of the study has been done on multichannel signal processing. The electromyogrphic signals from the multi-channel data acquisition system will increase the classification efficiency with the increase in classification accuracy but with increase of diminishing effect in signals if the number of channels is increase to 4 or more [18].

Many researchers have chosen multi-channel that is multiple electrode can be used to perform some specific function with only designated electrodes but some want to move further ahead by leaving aside this strategy. Furthermore the number of classes can be increased to increase the classification accuracy. It is understood that the accuracy will decrease because of the nature of accuracy and when the output data flowing through different channels increase which affects the quality of signal by affecting its feature space. Therefore increasing number of channels will certainly affect its feature space assimilated with each class [19].

A back-propagation neural network is the solution of the discussed problem, in which the EMG signal are acquired for different hand movement earlier defined which can be flexion, extension, supination and pronation or else. Calculated Time frequency based parameters can be used as input to this classifier, Which can be Wavelet transform, Moving Average, Auto regression, Root Mean Square, Fast Fourier Transform, Variance, Standard deviation, Slope Sign Change, Willson Amplitude, Zero crossing, Wave Length. Selection of these features is the most important part of neural theory. Selecting relevant features gives the pattern which then can further be easily classified.

\section{B. $K$ Nearest Neighbor Classifier}

Neural Network classifier is considered as slow and time consuming. $\mathrm{K}$ nearest neighbor can be used to get accurate results in span to time. In this classification technique, the reference vectors from all the required motions can be used to calculate the distance between the input vectors of present state [20].

KNN first assigns class to the un-known events which represents majority of its nearest neighbors. Assignment of class is based on most suitable pattern nearest to the system measured on the basis of Eculidean distance. Labeling is done to classify segment that is most frequently represented among the $\mathrm{K}$ nearest neighbor. At the end decision is made on the basis of taking a vote and by examining the labels. Discriminative approach been employed in KNN which is more suitable when reliable probabilistic densities are difficult to find. 


\section{EXPERIMENTATION AND PROCEDURE}

Acquired signal from the electrodes have frequency that ranges between $0-500 \mathrm{~Hz}$ which have noise from different sources such as cross talk, artifacts and above all power line sources. These noise ranges from $50-60 \mathrm{~Hz}$ which has to be removed before amplification is done. Signal is acquired from two electrodes which are $1-2 \mathrm{~cm}$ apart from each other; the signal common to both is rejected with the help of differential amplifier. The amplitude of the acquired signal ranges from 0 $20 \mathrm{mV}$.

Amplification is one of the most important steps in active prosthesis done with the help of instrumentation amplifier; this amplification can be achieved with INA 121. It is an IC which has vast applications in biomedical field, which has ability to amplify up to 10,000 times [21]. Once the detection of EMG signals is achieved through sensing electrodes, differential technique is employed with the help of operational amplifier to achieve first step in amplification [22].

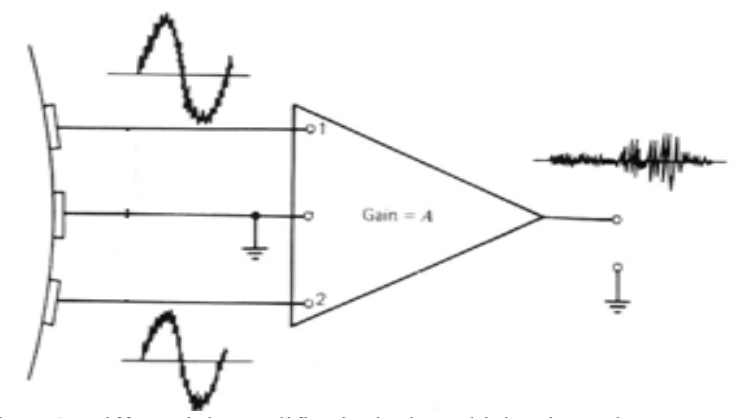

Figure3. Differential Amplifier is device which rejects the common signal of both the inputs provided to it.

Acquired analog signal whose frequency ranges form 50$200 \mathrm{~Hz}$ and amplitude varies form 0-5 volt obtained after carrying out the above mentioned procedure. This analog signal has to be converted in to digital signal with the help of Analog to Digital Converter (ADC) which is commonly used in modern electronics. During digitization following things have to be kept in mind resolution, range of conversion and the sampling rate. The maximum voltage which an ADC can convert in to digital format is known as range of conversion. Sampling rate is kept high for the minimum loss of data; dynamic range of conversion is kept high which keep the amplification output small.

In the study 16 bit ADC has been used which come as a peripheral with ATMEGA16 microcontroller [23]. This has multiple channels with on chip-2 cycle multiplier. Above information completely fulfill our requirement for amplified signal between 1-4.8 volts. As flexion and extension of hand take place, signal after amplification is put into mentioned microcontroller for further digitization and motor control. ADC convert amplified analog signal it to digital format, where reference of the ADC is given 5 volts.

Thresholding technique is applied for the controlling different movements. Peak values are measured from each targeted muscle and threshold value is selected, threshold value must be 2 volts less than the peak value in case of flexion and extension. As the targeted muscles fire, the motor unit get excited, signal detected by the gelled electrodes, preamplification is done with differential amplifier, noise is reduced by using high pass filter, then the amplification is done with mentioned instrumentation amplifier, ADC convert this amplified analog signal in to digital signal which is between $0-5$ volts and send it further for processing.

Comparison is being done between threshold value and the system value, as it passes the threshold value it gives the output as 1 which moves the motor in the desired direction. Same procedure can be repeated for desired motion of supination and pronation by assigning different motor points on the targeted muscle. With accurate placement of electrodes, we can able to differentiate between the motions for all the fingers, hence giving more functionality as well degree of freedom to the patient. Here are some experimental results after finger classification being done with the help of gelled electrodes.

\begin{tabular}{|c|l|c|c|}
\hline S.No & $\begin{array}{l}\text { Fingers and } \\
\text { Hand } \\
\text { movements }\end{array}$ & $\begin{array}{l}\text { Acquired peak } \\
\text { voltage level } \\
\text { before } \\
\text { contracting }\end{array}$ & $\begin{array}{l}\text { Acquired peak } \\
\text { voltage level } \\
\text { after } \\
\text { contracting }\end{array}$ \\
\hline 1 & Hand Flexion & $0.16 \mathrm{~V}$ & $4.8 \mathrm{~V}$ \\
\hline 2 & $\begin{array}{l}\text { Hand } \\
\text { Extension }\end{array}$ & $0.12 \mathrm{~V}$ & $4.6 \mathrm{~V}$ \\
\hline 3 & $\begin{array}{l}\text { Index finger } \\
\text { Flexion }\end{array}$ & $0.5 \mathrm{~V}$ & $1.8 \mathrm{~V}$ \\
\hline 4 & $\begin{array}{l}\text { Thumb } \\
\text { Flexion }\end{array}$ & $0.6 \mathrm{~V}$ & $3.8 \mathrm{~V}$ \\
\hline 5 & $\begin{array}{l}\text { Ring finger } \\
\text { Flexion }\end{array}$ & $0.2 \mathrm{~V}$ & $3.1 \mathrm{~V}$ \\
\hline
\end{tabular}

TABLE: VOLTAGE LEVELS AFTER AMPLIFICATION

Table shows different voltage levels from different muscles of hand and fingers which were explained earlier, these signals were acquired after amplification, we can set the threshold between these two peak values for which the motor can be derived for the predefined value, that value should be nearer to peak value contracting value.

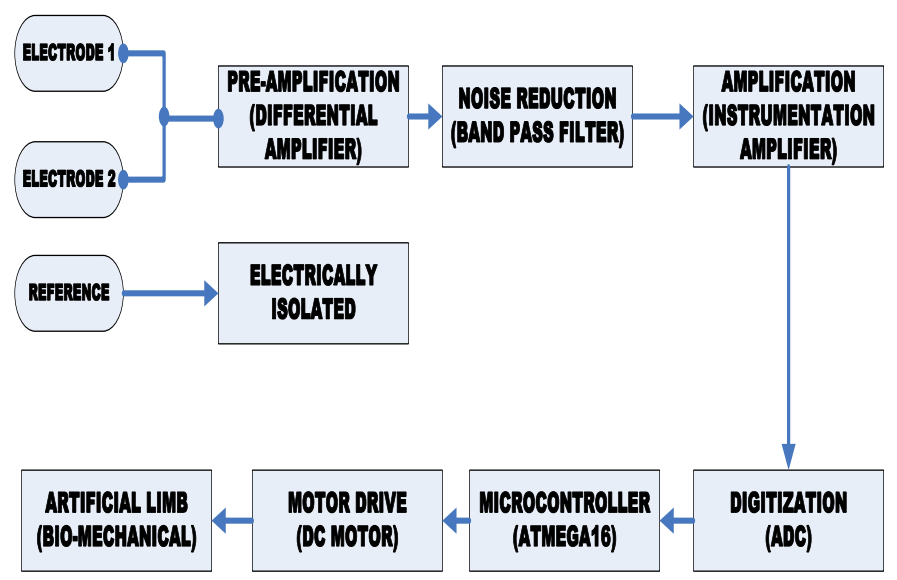

Figure4. Block diagram indicating all step involved in active prosthesis 
The amplitude of EMG signal emanating from belly of the muscles varies person to person. A muscular man can emanate EMG signal of more amplitude than a normal man. Acquisition of superimposed signals, amplitude from the contracting muscles will be higher as compared to isolated muscle that is valid in case of flexion and extension. It will have low amplitudes when placed on the dedicated muscle for each fingers. The given study is taken on normal built.

\section{CONCLUSION}

A novel method for flexion and extension of mechanical arm is successfully achieved with the help of EMG. Electrode placement on the targeted muscle for the clean signal is necessary. During study it was revealed that each muscle form a set pattern of signal whenever the flexion or extension take place. Extracting the statistical features from EMG signals for classifying the motions through described classifiers, different wavelet function can be used for enhancing the classification rate. Amplitude of EMG signal varies as number of muscles fire increase or decrease. This study is a step forward towards achieving active prosthesis which is not only light weight, cost effective but also a successful replacement of upper limb amputation. Noise reduction techniques have been emphasized for future work.

\section{REFERENCES}

[1] A.G. Outten, S.J. Roberts and M.J. Stokes "Analysis of human muscle activity", Artificial Intelligence Methods for Biomedical Data Processing, IEEE Colloquium, London, 26 April,1996.

[2] M.L. Harba and G.E. Chee "Muscle Mechanomyographic and electromyographic signals compared with reference to action potential average propagation velocity", Engineering in Medicine and Biology Society, $19^{\text {th }}$ Annual International Conference of the IEEE, Vol.3, and 6th August 2002.

[3] G. Matrone, C. Cipriani, M. C. Carrozza and G. Magenes "Two-Channel Real-Time EMG control of a Dexterous Hand Prosthesis", $5^{\text {th }}$ International IEEE EMBS Conference on Neural Engineering, Cacun, Mexico, April 27-May 1, pages: 554-557, 2011.

[4] G.W. Choi, G.H. Choe, I.H. Moon and M.S. Mun "Development of Surface Mayoelectric Sensor for Mayoelectric Hand Prosthesis", Power Electronics Specialists Conference, 2006. PESC '06. $37^{\text {th }}$ IEEE, Pages 1-5, 2006.

[5] P. Zhu "Design of Surface Electromayography Detection Circuit", International Conference on Future Information Technology and Management Engineering, volume.1, pages: 459-462, 2010.

[6] S. Shahid, J. Walker "Application of higher order statistics techniques to EMG signal to characterize the motor unit action potential",IEEE Transactions on Bio-medical Engineering, Vol.52, NO.7,July 2005.

[7] C.J. De Luca "Electromayography Encyclopedia of Medical Devices and Instrumentation", (John G. Webster Ed), John Wiley Publisher, 2006.

[8] C.N. Huang, C.H. Chen and H.Y. Chung "The Review of Applications and Measurements in Facial Electromayography", Journal of Medical and Biological Engineering, Vol.25, $23^{\text {rd }}$ November, 2004.

[9] Dr. Scott Day "Important Factors in Surface EMG Measurement", Bortec Biomedical Incorporated.

[10] N. Masso, F. Rey, D. Romero, G. Gual, L. Costa and A. German "Surface Electromayography and Applications in Sport" Apunts Medicina De L'Esport, Vol.45:127-136, February 5, 2010.

[11] C.J. De Luca "Surface Electromayography: Detection and Recording", Delsys Incorporated, 2002.
[12] S. Yacuab, P.Y. Gumery, K. Raoof "A Novel Signal Processing Method for Multi-electrode Surface Electromayography", Engineering in Medicine and Biology Society. Engineering Advances: New Opportunities for Biomedical Engineers, Proceedings of the $16^{\text {th }}$ Annual International Conference of the IEEE, Vol.2, pages 1336-1337, 1994.

[13] M. Malboubi, F. Razzazi, Ma. Sh, A. Davari "Power Line Noise Elimination from EMG Signals Using Adaptive Laguerre Filter with Fuzzy step size", $17^{\text {th }}$ Iranian Conference of Biomedical Engineering, pages $1-4,2010$.

[14] R.L. Ortalon, R.N. Mori, R.R. Pereira, C.M.N. Cabral, J.C. Pereira, J.A Cliquet "Evalution of Adaptive/Nonadaptive Filtering and Wavelet Transform Techniques for Noise Reduction in EMG Mobile Acquisition Equipment", Transactions on Neural Systems and Rehablitation Engineering, IEEE, Issue: 1, pages 60-69, 2003.

[15] F. Sebelius, M. Axelsson, N. Danielsen, J. Schouenborg, and T. Laurell, "Real Time Control Virtual Hand", Technology And Disability", vol.17 no. 3, pages 131-141, 2005.

[16] J. Pons, R. Ceres, E. Rocon, S. Levin, I. Markovitz, B. Saro, D. Reynaerts, W.V. Moorleghem and L. Bueno,"Virtual Reality Training and EMG Control of the MANUS Hand Prosthesis", Robotica, vol.23, no.03, pages 311-317, 2005.

[17] F.E.R. Mattioli, E.A. Lamounier, A. Cardoso, A.B. Soares, A.O. Andrade."Classification of EMG signals using Artificial Neural Networksfor Virtual hand Prosthesis Control", Annual International Conference of the IEEE, Engineering in Medicine and Biology Society, Pages 7254-7257, 2011.

[18] G. Tsenov, A.H. Zeghbib, F. Palis, N. Shoylev and V. Mladenov "Neural Networks for Online Classification of Hand and Finger Movements using Surface EMG signals", in Neural Networks Applications in Electrical Engineering, 2006,NEUREL $2006.8^{\text {th }}$ Seminar, pages 167-171, 2007.

[19] M.R. Ahsan, M.I. Ibrahimy, O.O. Khalifa "EMG Motion Pattern Classification through Design and Optimization of Neural Network", International Conference on Biomedical Engineering, ICOBE, pages 175-179, 2012.

[20] P. Geethanjali, K.K. Ray, P.V. Shanmuganathan "Actuation of Prosthetic Drive Using EMG”, TENCON 2009, IEEE Region 10 Conference, pages 1-5, 2009.

[21] Datasheet INA121 "FET input Low Power Instrumentation Amplifier", Burr-Brown Incorporated.

[22] Y. Shimomura, K. Iwanaga, H. Harada and T. Katsuura "Evaluation and Design of a Small Portable EMG Amplifier". Volume: 18, 61-67, 1999.

[23] Datasheet ATMEGA16 "8-bit AVR microcontroller with 16kBytes InSystem Programmable Flash”, ATMEL Incorporated.

\section{Creative Commons Attribution License 4.0 (Attribution 4.0 International, CC BY 4.0)}

This article is published under the terms of the Creative Commons Attribution License 4.0

https://creativecommons.org/licenses/by/4.0/deed.en_US 